\title{
СХЕМА ОРГАНИЗАЦИИ ВНУТРЕННЕГО ФИНАНСОВОГО КОНТРОЛЯ В РАМКАХ РАСХОДОВАНИЯ СРЕДСТВ НА ФЕДЕРАЛЬНЫЕ ПРОЕКТЫ
}

\author{
(C) 2021 Петров Александр Михайлович \\ доктор экономических наук, профессор Департамента бизнес-аналитики \\ Финансовый университет при Правительстве Российской Федерации, Россия, Москва \\ профессор кафедры бухгалтерского учета и налогообложения \\ Российский экономический университет им. Г. В.Плеханова, Россия, Москва \\ E-mail: palmi@inbox.ru
}

Эффективное исполнение федеральных проектов, в части расходования выделенных на них средств, опирается на оперативный и превентивный инструментарий внутреннего финансового контроля. В статье осуществление внутреннего финансового контроля в части расходования бюджетных средств, выделенных на федеральные проекты, рассматривается как со стороны распорядителей бюджетных средств, так и со стороны получателей этих средств.

Ключевые слова: бюджетные нарушения, внутренний финансовый контроль, федеральный проект, отраслевой риск, ключевые индикаторы риска.

Повышение эффективности и установление законности исполнения федеральных проектов, в части расходования выделенных на них средств, опирается на оперативный и превентивный инструментарий внутреннего финансового контроля (ВФК). Осуществление внутреннего финансового контроля в части расходования бюджетных средств, выделенных на федеральные проекты, можно рассматривать с двух основных позиций: распорядителей бюджетных средств и получателей этих средств (рисунок 1). Представим схему ВКФ в рамках расходования средств на федеральные проекты с позиции распределителей бюджетных средств (рисунок 2) [2].

В рамках этапа планирования федеральных проектов важная задача внутреннего финансового контроля, осуществляемого распоряди- телями средств - это недопущение противоправного или нерационального использования бюджетных средств.

На этапе планирования должна проводиться оценка и экспертиза проекта, в процессе которой определяется целесообразность решения проблемы проектно-целевым методом, соотносится ресурсное обеспечение исполнения проекта с планируемыми результатами, оценивается прогнозная эффективность проекта.

На этапе финансирования внутренний финансовый контроль, осуществляемый распорядителями бюджетных средств, должен быть направлен на оценку рациональности выделения средств и соизмерение расходов по реализации федерального проекта с ожидаемой эффективностью, положительным влиянием на экономи-

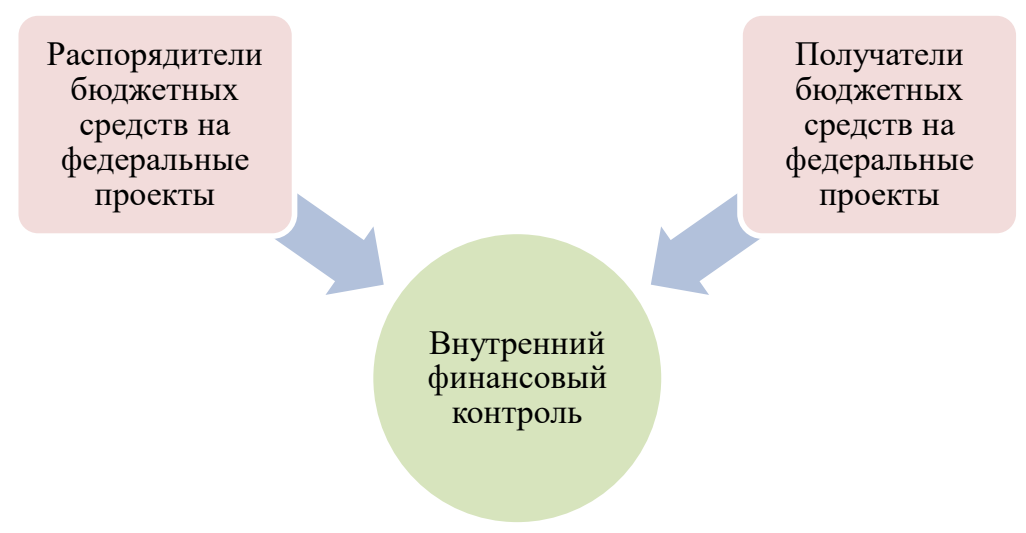

Puc. 1. Внутренний финансовый контроль распорядителей бюджетных средств на федеральные проекты и их получателей 


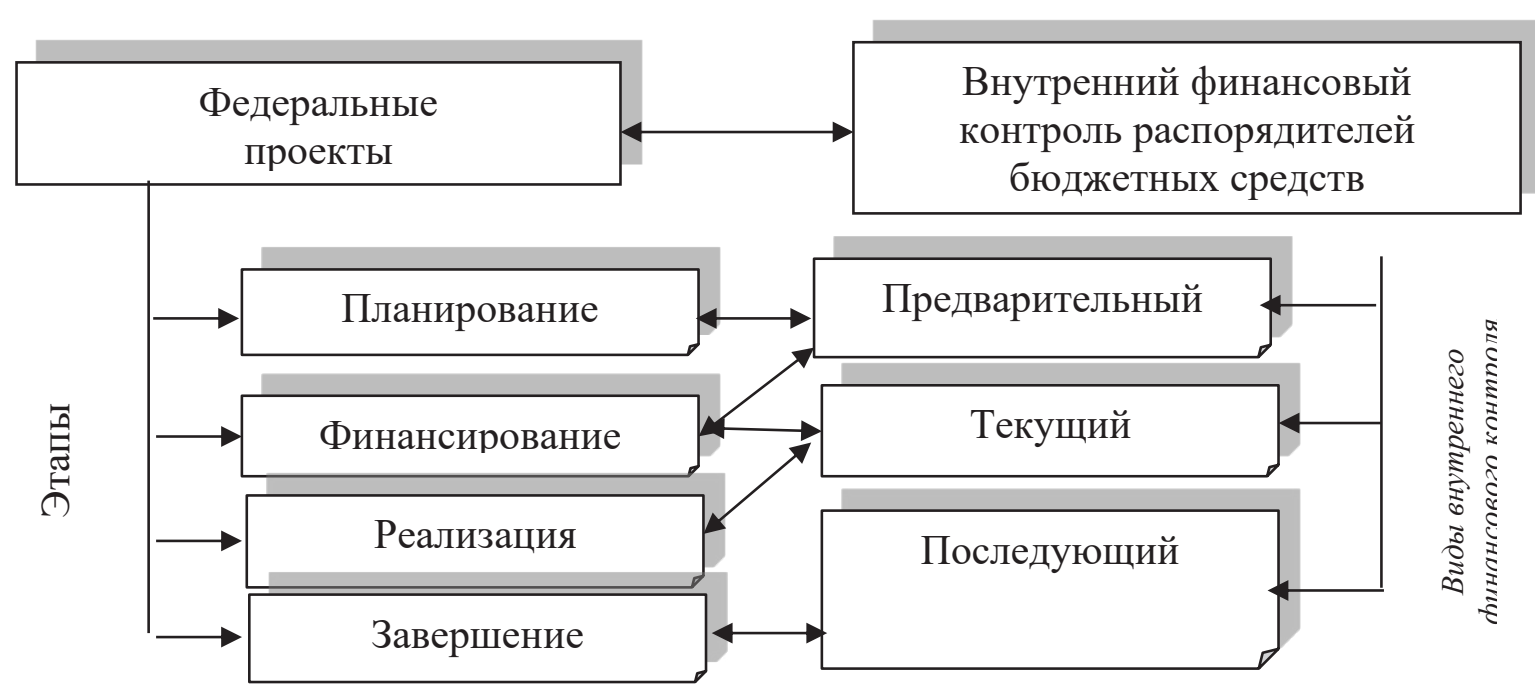

Puc. 2. Схема внутреннего финансового контроля в рамках расходования средств на федеральные проекты с позиции распределителей бюджетных средств

ческую, социальную сферу, научно-технический прогресс и иные макросферы. Схема внутреннего финансового контроля в рамках расходования средств на федеральные проекты с позиции распределителей бюджетных средств представлена на рисунке 2.

Кроме этого, необходимо оценить соответствие ключевых направлений федеральных проектов документам государственного стратегического планирования Российской Федерации.

Проверку обоснованности финансирования федерального проекта осуществляют с помощью предварительного контроля. Однако текущий контроль позволяет проверять процесс финансирования проектов на соответствие плановым графикам и его фактического осуществления, объемам, которые заявлены в рамках конкретных проектов.

На этапе реализации федеральных проектов реализуется текущий финансовый контроль распорядителями бюджетных средств, который направлен на проверку своевременности их выполнения и целевой направленности расходования бюджетных средств. На данном этапе в процессе проверки должны использоваться различные контрольные, экспертные, аналитические мероприятия по оценке промежуточной отчетной документации, платежных документов, подтверждающих расходные операции, связанные с бюджетными средствами.

На этапе реализации внутренний финансовый контроль позволяет: выявить нарушения; оперативно отреагировать устранением на выявленные нарушения, неточности, ошибки; скорректировать отдельные индикаторы и параметры проектов; уточнить ключевые показатели проектов; скорректировать затраты по мероприятиям федеральных проектов; скорректировать механизм реализации федеральных проектов. Но при этом следует понимать, что в большинстве случаев, корректировки различных показателей могут не привести к корректировке объемов финансирования проектов.

На этапе завершения федеральных проектов должен осуществляться последующий внутренний финансовый контроль распорядителями средств, ориентированный на проверку: целесообразности использования бюджетных средств; целевой направленности расходования бюджетных средств; наличия подтверждающих документов по расходованию бюджетных средств; эффективности расходования средств, предусмотренных проектами.

На этапе завершения федеральных проектов распорядителями средств могут выявляться нарушения, недостатки финансирования, финансовые махинации, а также привлекаться к ответственности виновные лица. Таким образом, каждый этап работы с федеральными проектами от момента планирования до его завершения требует детальной проверки. Это подтверждает необходимость разработки действенного оперативного и превентивного инструментария внутреннего финансового контроля в рамках 
расходования средств на федеральные проекты с позиции распределителей бюджетных средств [2].

На рисунке 3 отразим схему ВФК в части расходования средств, выделенных на федеральные проекты, с позиции получателя бюджетных средств.

Далее рассмотрим схему ВФК в части расходования средств, выделенных на федеральные проекты, с позиции получателей бюджетных средств.

На этапе участия должен применяться предварительный внутренний финансовый контроль, осуществляемый получателями средств, который направлен на оценку возможности исполнения федерального проекта и целевого использования средств. Кроме этого, на данном этапе необходимо выявить потребность в привлечении внешних соисполнителей, субподрядчиков или поставщиков, согласовать стоимость их работ, услуг в рамках выполнения заданий федеральных проектов. Требуется провести контроль заполнения необходимой документации на предмет законности, правильности и своевременности.

На этапе получения финансирования должен осуществляться текущий внутренний контроль получателями средств, направленный на проверку соблюдения графика поступления средств в рамках документации федеральных проектов, объема поступивших ресурсов.

На этапе реализации федеральных проектов получателями средств должен реализовываться текущий ВФК, направленный на проверку: соблюдения требований законодательства в рамках исполнения федеральных проектов; соблюдения условий контрактов в рамках исполнения федеральных проектов; соблюдения графика выполнения проектов; целевого расходования полученных средств; наличия всей подтверждающей документации по расходованию полученных бюджетных средств; правильности оформления всей подтверждающей документации по расходованию полученных бюджетных средств; целесообразности привлечения внешних соисполнителей, субподрядчиков или поставщиков; разумности и объективности стоимости работ, услуг привлеченных внешних соисполнителей, субподрядчиков или поставщиков в рамках выполнения заданий федеральных проектов; своевременности расчетов с привлеченными внешними соисполнителями, субподрядчиками или поставщиками в рамках выполнения заданий федеральных проектов; правильность оформления документации по сданным работам, выполненным услугам сторонними соисполнителями, субподрядчиками или поставщиками в рамках выполнения заданий федеральных проектов; своевременности расчетов с контрагентами по операциям, связанным с расходованием средств по выполнению федеральных проектов; правильности начисления налогов и их уплаты в рамках исполнения федеральных проектов; своевременности предоставления промежуточной отчетной документации по выполнению федеральных проектов.

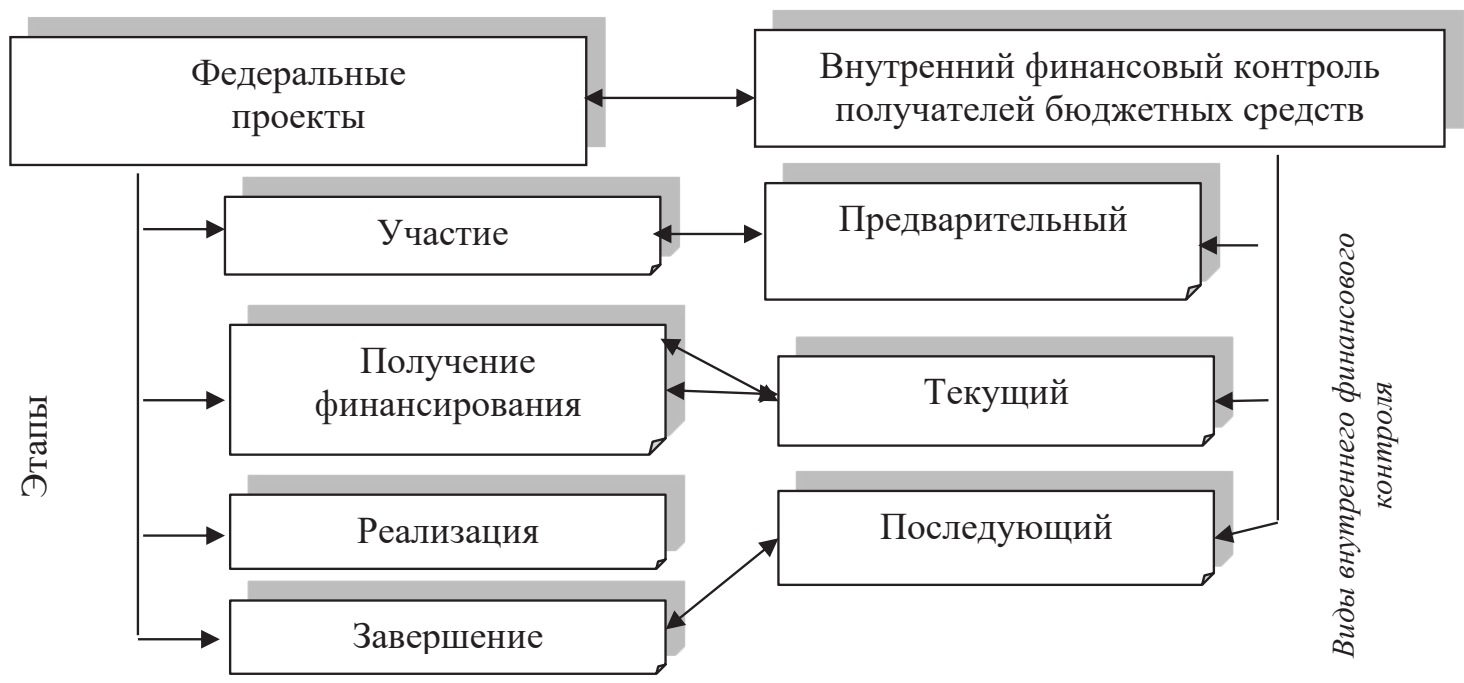

Рис. 3. Схема внутреннего финансового контроля в рамках расходования средств на федеральные проекты с позиции получателей бюджетных средств 
На этапе реализации в ходе внутренней проверки получателями средств необходимо применять в комплексе разные процедуры: контрольные, экспертные, аналитические. На данном этапе текущий внутренний финансовый контроль позволяет выявить хищения, финансовые мошенничества, сговоры с аффилированными лицами, иные правонарушения. Своевременное выявление недочетов и нарушений позволяет оперативно разработать мероприятия по их ликвидации, исправлению, и последующему недопущению.

На этапе завершения федеральных проектов получателями средств должен проводиться последующий внутренний финансовый контроль, ориентированный на проверку: соблюдения законодательства и требований контракта по сдаче отчетной документации по федеральным проектам; полного выполнения всех условий федеральных проектов; целевого расходования бюджетных средств; наличия подтверждающих документов по расходованию бюджетных средств; эффективности расходования средств, предусмотренных проектами.

На этапе завершения федеральных проектов получателями средств могут выявляться нарушения, мошеннические схемы, а также привлекаться к ответственности виновные лица. Таким образом, каждый этап работы с федеральными проектами от момента участия до его завершения требует регулярной и тщательной внутренней проверки, что подтверждает потребность в разработке эффективного оперативного и превентивного инструментария внутреннего финансового контроля в рамках расходования средств на федеральные проекты с позиции получателей бюджетных средств. Несмотря на разнообразие инструментов внутреннего финансового контроля в их основу должны ложиться оперативный и превентивный подходы [2].

\section{Библиографический список}

1. Петров А. М., Бурцева К. Ю. К вопросу организации внутреннего финансового контроля в рамках расходования средств на федеральные проекты // Экономические науки. - 2020. - № 188. - С. 113-119.

2. Петров, А. М. Оперативный и превентивный инструментарий реализации внутреннего финансового контроля в рамках расходования средств на федеральные проекты // Экономические науки. -2020 . - № 185. C. $169-178$.

3. INTERNAL CONTROL MANUAL FOR FEDERAL GRANTS [Электронный ресурс]. - URL: https://www.ipsk12.net/ cms/lib/MA02212674/ Centricity/Domain/275/IPS\%20MANUAL\%20FOR\%20FEDERAL\%20GRANTS-Final\%20 Version\%205.4.17.pdf (дата обращения: 10.03.2020).

4. Петрова О.А. «Бухгалтерский учет обеспечения исполнения контрактных обязательств в России» / Экономические науки 2021, № 9, стр. 232-234

5. Петрова О.А. «Цифровизация консолидированной финансовой отчетности в страховом бизнесе» / Экономические науки 2021, № 10

6. Петрова О.А. «Проблемы развития рынка факторинговых услуг» / Экономические науки 2021, № 10

7. Chen T.-C., Zhu W.-W., Jiao Z.-K., Petrov A.M. Creep-fatigue lifetime estimation of snagcu solder joints using an artificial neural network approach / Mechanics of Advanced Materials and Structures. 2021.

8. Shabbir M.S., Siddiqi A.F., Yapanto L.M., TonkovE.E., PoltarykhinA.L., PilyuginaA.V., PetrovA.M., Foroughi A., Valiullina D.A. Closed-loop supply chain design and pricing in competitive conditions by considering the variable value of return products using the whale optimization algorithm / Sustainability. 2021. T. 13. № 12.

9. Kavitha M., Mahmoud Z. H., Kishore K. H., Petrov A. M., Lekomtsev A., Iliushin P., Zekiy A. O., Salmani M. Application of Steinberg model for vibration lifetime evaluation of SN-AG-CU-based solder joints in power semiconductors / IEEE Transactions on Components, Packaging and Manufacturing Technology. 2021. T. 11. № 3. C. 444-450.

10. Shnaider V.V., PipkoE.G., PetrovA.M. Current information needs of passenger and freight transportation enterprises in Russia / Вестник Национальной академии наук Республики Казахстан. 2020. № 4 (386). С. $348-$ 353.

11. Petrov A., Tolmachev M., Shlychkov D., Basova M., Turishcheva T. Analysis of current trends of employment diversification at the international level: case study of pharmacy industry / Systematic Reviews in Pharmacy. 2020. T. 11. № 12. C. $1383-1390$. 